\title{
Identifying balance impairments in people with Parkinson's disease using video and wearable sensors
}

Article

Accepted Version

Creative Commons: Attribution-Noncommercial-No Derivative Works 4.0

Stack, E., Agarwal, V., King, R., Burnett, M., Tahavori, F., Janko, B., Harwin, W., Ashburn, A. and Kunkel, D. (2018) Identifying balance impairments in people with Parkinson's disease using video and wearable sensors. Gait \& posture, 62. pp. 321-326. ISSN 1879-2219 doi:

https://doi.org/10.1016/j.gaitpost.2018.03.047 Available at https://centaur.reading.ac.uk/76626/

It is advisable to refer to the publisher's version if you intend to cite from the work. See Guidance on citing.

To link to this article DOI: http://dx.doi.org/10.1016/j.gaitpost.2018.03.047

Publisher: Elsevier

All outputs in CentAUR are protected by Intellectual Property Rights law, including copyright law. Copyright and IPR is retained by the creators or other copyright holders. Terms and conditions for use of this material are defined in the End User Agreement. 


\section{CentAUR}

Central Archive at the University of Reading

Reading's research outputs online 


\title{
Identifying balance impairments in people with Parkinson's disease using video and wearable sensors
}

\author{
Emma Stack ${ }^{\mathrm{a}}$, Veena Agarwal ${ }^{\mathrm{a}, \mathrm{c}}$, Rachel King ${ }^{\mathrm{b}}$, Malcolm Burnett ${ }^{\mathrm{a}}$, Fatemeh \\ Tahavori $^{a}$, Balazs Janko ${ }^{b}$, William Harwin ${ }^{b}$, Ann Ashburn ${ }^{a}$, Dorit Kunkel ${ }^{a}$ \\ a Faculty of Health Sciences, University of Southampton, Southampton, UK \\ b School of Systems Engineering, University of Reading, Reading, UK \\ c Southampton Centre for Biomedical Research, University Hospital Southampton NHS Foundation \\ Trust.
}

\section{Corresponding Author:}

Dr Dorit Kunkel, Mailpoint 886, Southampton General Hospital, Tremona Road, Southampton SO16 6YD, UK; emaildh197@soton.ac.uk

\section{Word count (Introduction-Conclusions): $\mathbf{3 0 0 0}$}

\section{Acknowledgements}

This work was performed under the SPHERE IRC funded by the UK Engineering and Physical Sciences Research Council (EPSRC), Grant EP/K031910/1. The authors would like to thank the members of Parkinson's UK and the University of the Third Age (U3A) who participated. 


\begin{abstract}
Background: Falls and near falls are common among people with Parkinson's (PwP). To date, most wearable sensor research focussed on fall detection, few studies explored if wearable sensors can detect instability.
\end{abstract}

Research question: Can instability (caution or near-falls) be detected using wearable sensors in comparison to video analysis?

Methods: Twenty-four people (aged 60 - 86) with and without Parkinson's were recruited from community groups. Movements (e.g. walking, turning, transfers and reaching) were observed in the gait laboratory and/or at home; recorded using clinical measures, video and five wearable sensors (attached on the waist, ankles and wrists). After defining 'caution' and 'instability', two researchers evaluated video data and a third the raw wearable sensor data; blinded to each other's evaluations. Agreement between video and sensor data was calculated on stability, timing, step count and strategy.

Results: Data was available for 117 performances: 82 (70\%) appeared stable on video. Ratings agreed in 86/117 cases (74\%). Highest agreement was noted for chair transfer, timed up and go test and 3m walks. Video analysts noted caution (slow, contained movements, safety-enhancing postures and concentration) and/or instability (saving reactions, stopping after stumbling or veering) in 40/134 performances (30\%): raw wearable sensor data identified $16 / 35$ performances rated cautious or unstable (sensitivity $46 \%$ ) and $70 / 82$ rated stable (specificity $85 \%$ ). There was a $54 \%$ chance that a performance identified from wearable sensors as cautious/unstable was so; rising to $80 \%$ for stable movements. Significance: Agreement between wearable sensor and video data suggested that wearable sensors can detect subtle instability and near-falls. Caution and instability were observed in nearly a third of performances, suggesting that simple, mildly challenging actions, with clearly defined start- and end-points, may be most amenable to monitoring during freeliving at home. Using the genuine near-falls recorded, work continues to automatically detect subtle instability using algorithms. 


\section{Introduction}

People with Parkinson's (PwP) fall twice as often as healthy older people [1-3]; 75\% PwP reported near-falls in one year [4]. Fall prevention is a healthcare priority; programmes in PwP improve balance, although few demonstrated a reduction in fall rates $[3,5-8]$.

Monitoring falls and instability could be beneficial for inactive people in poor health who fall frequently and sustain more injuries than healthy active adults [9-10].

Interest in automated fall detection is growing [11]. Although falls are unintentional, to date wearable sensor fall detection studies often focussed on simulated falls [12]. Only 7\% of wearable sensor reports included monitoring in 'a real-world setting' [13]; few researchers recorded natural falls [14-15]. Wearable sensors revealed differences between fallers and non-fallers with Parkinson's and correlations with other measures of fall risk, such as the Timed Up and Go Test (TUG), [16]. They have predicted time to first fall (from gait variability) better than traditional measures [17] and the response to a change in medication [18]. In one study, researchers provoked PwP into taking missteps by asking them to perform a protocol of increasingly challenging balance tasks in a laboratory [19]. Misstep detection was calculated by dividing the number of sensor detected missteps by the number of missteps identified by clinicians. The algorithm achieved a 'Hit ratio' of $93 \%$ and was used to detect 'suspected missteps' in the home environment. Missteps were reported among a higher proportion of fallers than non-fallers but there was no way of establishing sensitivity or specificity [19].

Interest in 'cautious gait' [20-24] is growing. Observational gait analysis suggests elderly people with 'cautious gait' walk slowly, taking short strides [13 - 14], appear unsteady [20] and reduce the velocity of their centre of mass [21]. Yet despite recognising 'traditional tools' in evaluating fall risk could be augmented [19], few researchers have attempted to detect caution or near-falls automatically [19, 25-26].

Automatic detection of subtle instability could provide an opportunity to intervene at the near-falls [4] stage. Therefore the aim of this study was to explore the use of wearable sensors, in comparison to expert clinician video analysis, to detect subtle instability (caution or near-falls). For this study, the clinically based ground truth was the identification of 'subtle instability' made by the movement analysts (interpreting movement, facial 
expressions and comments) from video, cross checked with the raw data from wearable sensors (accelerometer and gyroscope).

\section{Methods}

Ethical approval was obtained by the University of Southampton, Faculty of Health Sciences. The researcher contacted Parkinson's UK (a UK charity) in Southampton, Reading and Newbury as well as the University of the Third Age (a UK organisation providing educational and leisure activities to retired and semi-retired individuals). With branch chair approval, the study was publicised in newsletters and branch meetings. We invited participation by leaving information packs with freepost envelopes with group chairs. Those who were able to provide consent, walk unaided indoors, follow simple instructions and perform mobility tests for one hour were eligible to take part. Twenty-four people, 10 PwP (mean age 74 (SD 7), Hoehn and Yahr 1-5, 6 female) and 14 healthy adults (mean age 74, 13 female) returned the completed reply slip indicating their interest to take part and were subsequently recruited to the study.

Their movements were observed at Southampton General Hospital gait laboratory and/or at home performing actions associated with falling [9], recording with a tripod-mounted HD video camera (at 25 frames/second) and five unobtrusive (wristwatch size) wearable sensors. The battery-powered, non-commercial, tri-axial wearable sensors containing accelerometers ( $\pm 8 \mathrm{~g}$ range at $0.25 \mathrm{mg}$ resolution) and gyroscopes $\left(2000^{\circ} /\right.$ second at $0.06 \mathrm{dps}$ resolution) logged accelerations and angular velocities for subsequent downloading and analysis. The researchers recorded age, gender, height, weight, medical history and recent falls. They started the camera, activated and attached wearable sensors on Velcro straps round the waist, ankles and wrists, and measured limb-lengths and sensor positions and instructed participants to perform:

- Chair Transfers (sit-to-stand and stand-to-sit).

- TUG [16]: From a chair with arms, "Stand, walk 3m, then return to sitting"

- Standing-Start $180^{\circ}$ Turn Test (SS-180), [27]: Facing away from the camera, turn freely left or right to walk toward it, then repeat but "turn in the opposite direction"

- 3m Walk [28]: "Walk 3m toward the camera" 
- Tandem Walk [29]: "Take 10 steps in-line toward the camera, so heel touches toe each time"

- Rising-to-Walk [30]: From sitting, "Rise to walk straight away (without pausing)"

- Reaching High and Low [31]: Touch a target above head height; pick a coin off the floor

Participants attempted the SS-180 (consisting of two turns) once, and other tests three times. Before and after each trial, participants waved to generate a recognisable pattern in the wearable sensors data, facilitating synchronisation and processing. For the purpose of the study considered a near-fall (near-miss) as an occasion on which individuals would have had a fall if they did not manage to save themselves [4].

The following questions guided analysis:

1. How do video analysts identify caution and near-falls during mobility tests?

2. Can eyeballing raw wearable data detect the caution and near-falls?

3. On which tests (and parameters) do video and wearable ratings agree most closely?

Blind to each other's evaluations, two researchers evaluated the videos and one the raw wearable sensor data using identical guidelines:

- Rate performance 'cautious' and/or 'unstable', respectively, if participants appeared concerned/ alarmed about their balance (making sudden movements or saving reactions) in at least one trial.

- Time tasks onset of movement until participant is sitting still again (chair transfers and TUG), until onset of first walking step (Rise-to-Walk), from onset of first turning step until onset of first walking step (SS-180); onset of first step until a foot crossed the $3 \mathrm{~m}$ line ( $3 \mathrm{~m}$ Walk); and onset of first step until end of tenth step (Tandem Walk).

- Rate transfers (chair, TUG and Rise-to-Walk) using the Parkinson Activity Scale (PAS), [32]: 4 = Normal, no apparent difficulty ( 2 if hands used); 3 = Mild Difficulty (toes dorsiflex, arms swing or 'rocks'; uncontrolled landing); 2 = Difficult, many tries, slow; abrupt landing ( 1 if hands used).

- Count SS-180 turning and 3m Walk steps

- Select 'Turn Type' [27]. 
- Score Tandem Walk 'deviations from a straight line' [29]: 0 = Normal for 10 steps; 1 = 1 -3 deviations; 2 = >3 deviations.

- Determine Rise-to-Walk 'Fluidity' (yes/no) from whether the participant moved smoothly from the transfer into walking without pausing [30].

- Rate the 'Use of Support' (yes/no) during high and low reaches, alongside high reach strategy ('flat feet' or 'up on toes'), and low reach strategy ('bend' from the waist or 'squat' bending the knees) [31].

The video analysts rated cautious and/or unstable movements together; grouped their comments and generate descriptions of apparent caution and/or instability. The wearable sensor analyst reviewed the sensor data for each activity, and based on the sensor output and activity performed, rated the trials. This was achieved by visually inspecting the accelerometer and gyroscope output of the most appropriate sensors as follows:

1. Performances were rated 'cautious' based on the time taken to perform activities and 'smoothness' in the accelerometer data, slower movements indicating caution, sudden or unexpected accelerations from the wrist, ankle and waist worn sensor data were rated 'unstable'.

2. Chair transfer, TUG and SS-180 were timed determining the beginning and end of movements from waist worn gyroscopes; timings for the $3 \mathrm{~m}$ and tandem walks were estimated from ankle worn sensors.

3. Chair transfers were assessed by exploring peaks in acceleration (and multiple attempts to stand) from the waist worn sensor. Symmetric and steady change in the accelerometer gravity vector from wrist sensors suggested use of hands (but could be confused with the hands resting on the knees or lap).

4. Turning step count was estimated from the ankle accelerometer and gyroscope data by examining the peaks in outputs.

5. Turn direction data was based on positive or negative rotation about the y-axis (primary torso axis) using waist worn gyroscope data; turn type inferred based on number of steps from the ankle worn sensors.

6. Tandem walk instability was rated by identifying high/sudden accelerations from wrists sensors (interpreted as balancing or 'saving' reactions), additional steps (seen in ankle sensors) were interpreted as balancing steps. 
7. The fluidity of the rise to walk was determined by detecting any pause between the sit-tostand phase (waist worn sensor) and the first steps (ankle worn sensor).

8. Indications of foot movements from ankle sensors during high reach indicated if participants came up onto their toes or if feet remained flat on the floor. Change in gravity vector data from waist worn sensors was used to estimate amount of waist flexion during low reaches.

The percentage agreement on stability between video and wearable sensors the sensitivity and specificity (performances rated unstable or stable from video or sensors) and positive and negative predictive values (chances of identifying instability and stability) were calculated. For continuous variables the mean difference between video and wearable timings and step counts and the 95\% limits of agreement (mean difference $+/-2$ standard deviations (SD)) are presented and to rank the tests and parameters on agreement, the $95 \%$ limit as a percentage of the video mean are presented were calculated.

\section{Results}

Twenty-four participants (19 women), 10 with Parkinson's, aged 60 to 86 years (mean 74 \pm 8 ), $1.5 \mathrm{~m}$ to $1.8 \mathrm{~m}$ tall (mean $1.6 \pm 0.1$ ), weighing $40 \mathrm{~kg}$ to $94 \mathrm{~kg}$ (mean $67 \pm 12$ ) took part. The sample comprised 12 people within normal weight range, eight overweight, one underweight and three obese (mean BMI $25 \pm 5$ ) participants. Five participants (four with Parkinson's) recalled repeated falls (median four). Fifteen people participated at home and 12 in the laboratory, including three in both settings. During home testing a Low Reach and 2 Rise-to-Walk were omitted for safety and 2 SS-180 and 5 TUG were omitted due to insufficient space. Sensor data was missing due to wearable failures for two laboratory participants (3\%), video data was missing due to recording errors for three home participants (2\%) and $1 \%$ of data was missing due to overlooked evaluations. Video analysts rated 25/134 performances (19\%) cautious only; seven (5\%) unstable only; eight (6\%) cautious and unstable; and 94 (70\%) neither. In the 33 cautious performances (25\%) participants concentrated markedly on the task $(n=18)$, making slow or contained movements $(n=27)$ in safety-enhancing postures $(n=22)$. In the 19 unstable performances 
$(14 \%)$ participants veered or counter-balanced $(n=10)$ taking errant steps or stumbles $(n=$ 12) necessitating saving reactions or stopping $(n=15)$.

Video and wearable sensor data on stability was available for 117 performances: 82 (70\%) appeared stable on video. Ratings agreed in $86 / 117$ cases (74\%) and highest agreement was noted for chair transfer, TUG and 3m walk, see Table 1. Wearable sensor data identified $16 / 35$ performances rated cautious or unstable from video (sensitivity $46 \%$ ), Table 2 , and $70 / 82$ stable performances (specificity $85 \%$ ). There was a $57 \%$ chance a performance using wearable sensors that was identified as cautious or unstable was cautious or unstable (16/28), and a 79\% chance a performance detected using wearable sensors identified as stable was stable (70/89).

The TUG generated closest agreement on time (Table 3), the $95 \%$ limit of agreement (-1.9s) being equivalent to $13 \%$ of the video mean (15.4s). However, balance performance of the TUG was relatively unchallenging as only $6 / 20$ participants (30\%) approached it with caution. In contrast, Rise-to-Walk time generated poor agreement on time, the $95 \%$ limit being $78 \%$ of the video mean $(2.2 \mathrm{~s} / 2.8 \mathrm{~s})$. However Rise-to-Walk generated most near-falls: $2 / 13$ participants (15\%) appeared unstable setting off to walk straight from rising and the test was omitted for two participants who deemed likely to fall.

Among other strategies (Table 4), best agreement (77\%) was on whether participants rose onto their toes during High Reach. Agreement on reaching strategies and stability varied because it was difficult to identify a dividing line between the strategies, even from video. Closest agreement was on whether standing participants rose onto their toes; simple plantarflexion enabled a higher reach but in a less stable position on a smaller base. Movements were more complex when participants bent from the waist or squatted to reach to the floor.

Agreement on SS-180 Turn Types was 66\% overall but there was zero agreement on 'Toward' and 'Pivotal' turns, while agreement for 'On-the-Spot' turns was 73\% and for 'Lateral' turns was 77\%. The $3 \mathrm{~m}$ Walk generated closer agreement on step count than the SS-180: for both tests, agreement was closer on timing than on step count. PAS generated 
better agreement on TUG and Rise-to-walk (21/31, 68\%) than on chair transfers (28/50, $56 \%$, Table 4): in 26/32 discrepancies (81\%) one of the wearable sensors used (in 20/26 cases) suggested the participant used their hands, while the other wearable sensor suggested they did not. Worst agreement (40\%) was on deviation from heel-toe gait during Tandem Walk. Video analysts noted many obvious saving reactions (including stepping wide of the intended line, raising the arms rapidly, or grabbing the handrail) during Tandem walking, rated everyone cautious and observed near-fall in 6/10 participants (60\%).

\section{Discussion}

Wearable sensor technology is advancing. As well as recording functional activities outside the laboratory, it is also vital to check validity of this data to ensure that the outputs are clinically relevant [33-34]. In the current study, movements during everyday activities were successfully recorded using video and wearable sensors for 24 older people in conjunction with clinically relevant gait and balance outcome measures. Only $6 \%$ of the potential data was lost due to technical and operator errors. In agreement with others, our findings demonstrated that with clear definitions, wearable sensors could detect subtle instability [34-36] in (79\%) cases when detecting caution and in $97(83 \%)$ cases when detecting nearfalls.

Three activities emerged with the highest agreement when identifying subtle instabilities: chair transfer, TUG and 3m walk. Similarly, there was good agreement in terms of smaller deviations from the video mean for TUG time and 3m walk time. However, during this preliminary exercise, the wearable sensor data lacked sensitivity (in contrast to previous research on fall detection, where the number of false alarms is high [37]).

Activities like TUG (i.e. incorporating multiple actions between clearly defined start- and end-points) and turning $180^{\circ}$ from a standing start is likely to cause instability, are probably well suited to monitoring in the home when attempting to detect subtle instability, e.g. the chair-to-stair route [38]. However, being small and naturally cluttered, the home environment does not lend itself well to video surveillance [38]; highlighting wearable sensors as a possible alternative. 
As optimum sensor placement positions for exploring instability has not yet been established [35] we used multiple sensor locations. Whilst most studies used waist sensors (85\%); others reported sensor placements on landmarks including the wrist [35]. As PwP generally have lower arm swing [39] wrist sensors might not ideal in this group of patients. However, as near-fall detection improves when multiple devices are worn [36], arm swing changes in PwP are linked to fall risk [40], and arm movements frequently used to regain stability [41] we including wrist sensors but based our analysis primarily on waist sensors when detecting gait instability [35-36].

Others 'provoked missteps' arguing that they 'occur infrequently and almost never in front of a clinician or in the laboratory' [19]. We disagree, arguing that 'provoked missteps' may differ from genuine near falls. Although we enhanced participant safety by having a researcher close throughout testing, participants appeared cautious and/or unstable in $40 / 134$ performances captured (30\%). We managed to capture genuine near falls in the present study, adding to the existing body of knowledge.

The features indicative of near-falls from video were all movement-related (veering; stumbling; and saving reactions) and the indicator of caution recognised most frequently was 'slow, contained movement'. Video analysts also noted 'marked concentration' (e.g. not speaking, fixed gaze, breathe holding) and 'safety-enhancing posture'. Previous research has highlighted similar features among people at risk of falling or moving cautiously, i.e. decreased arm swing [11], adapted visual sampling behaviour [42] and 'increased or variable walking base' [20].

One 'safety-enhancing posture', holding (or hovering near) support, indicated caution in the current study, as elsewhere [38]. However, wearable sensors were inaccurate in determining whether people supported themselves during transfers or reaching: we would not recommend monitoring 'support' in-home with sensors. As with Rise-to-Walk, agreement on chair transfer timing was poor; with such brief actions, any discrepancy appears significant. During free living, we recommend not timing transfers but monitoring stability instead; in the current study wearable sensors agreed best on detecting caution and instability during chair transfers. 
When rising, turning or walking merge, it was difficult to identify boundaries (which may lead to inaccurately discerning the activity let alone whether it is unstable) and a background dense in challenges can obscure instances of instability. Many discrepancies between ratings in the present study can be resolved when clinical and computer scientists together use relevant definitions and feature extraction (such as sensor pose angle over time, gross excursions of mean acceleration). Although they were working with the same definitions (e.g. of start- and end-points, strategies and instability), it is only through collaboration that ground truth can inform machine learning.

\section{Limitations}

We recruited participants who responded to invitations via local support groups. Convenience sampling has inherent vulnerabilities to selection bias and sampling error which limit generalisability of our findings. However, the main focus of our study was not to explore how different groups of people performed the tests but on the agreement between modes of quantifying movements using video and wearable sensors. We are confident that we obtained good quality data to answer our specific research question despite recruiting a convenience sample.

\section{Conclusion}

Agreement between wearable sensor and video data suggests that wearable sensors can detect subtle instability and might be a useful adjunct when exploring near-falls. We recommend including chair transfer, TUG and $3 \mathrm{~m}$ walk in future studies. Our assessment protocol generated caution and instability in nearly a third of performances. The 'instability' data generated is now being used to develop sensitive algorithms capable of detecting subtle instability among people at risk of falling.

\section{References}

[1] Lamont RM, Morris ME, Menz HB, McGinley JL, Brauer SG. Falls in people with Parkinson's disease: A prospective comparison of community and home-based falls. Gait Posture. 2017; 55: 62-7. 
[2] Pickering RM, Grimbergen YA, Rigney U, Ashburn A, Mazibrada G, Wood B, Gray P, Kerr G, Bloem BR. A meta - analysis of six prospective studies of falling in Parkinson's disease. Movement Disorders. 2007; 22: 1892-900.

[3] Canning CG, Paul SS, Nieuwboer A. Prevention of falls in Parkinson's disease: a review of fall risk factors and the role of physical interventions. Neurodegener Dis Manag. $2014 ; 4: 203-21$.

[4] Stack E, Ashburn A. Fall events described by people with Parkinson's disease: implications for clinical interviewing and the research agenda. Physiother Res Int. 1999; 4:190-200.

[5] Ashburn A, Fazakarley L, Ballinger $\mathrm{C}$ et al. A randomised controlled trial of an exercise programme for people with Parkinson's disease who repeatedly fall, J Neuro Neurosurg Psych. 2007; 78 : 678-84.

[6] Canning C, Sherrington C, Lord S, Close J, Heritier S, Heller G, Howard K, Allen N, Latt M, Murray S, O'Rouke S, Paul S, Song J, Fung V. Exercise for falls prevention in Parkinson's disease. Neurology. 2015; 84: 304-12.

[7] Morris M, Taylor N, Watts J, Evans A, Horne M, Kempster P, Danoudis M, McGinley J, Martin C, Menz A Home program of strength training, movement strategy training and education did not prevent falls in people with Parkinson's disease: a randomised trial. J Physiother. 2017; 63: 94-100.

[8] Morris M, Menz H, McGinley J, Watts MComm J, Huxham F, Murphy A, Danoudis M, lansek R A randomised controlled trial to reduce falls in people with Parkinson's Disease. Neurorehabil Neural Repair 2015; 29:777-85.

[9] Stevens JA, Mahoney JE, Ehrenreich H. Circumstances and outcomes of falls among high risk community-dwelling older adults. Inj Epidemiol. 2014; 1:5. doi: 10.1186/21971714-1-5.

[10] Cahill S, Stancliffe RJ, Clemson L, Durvasula S. Reconstructing the fall: individual, behavioural and contextual factors associated with falls in individuals with intellectual disability. JIDR. 2014; 58 :321-332. doi: 10.1111/jir.12015.

[11] Schwickert L, Becker C, Lindemann U, Maréchal C, Bourke A, Chiari L, Helbostad JL, Zijlstra W, Aminian K, Todd C, Bandinelli S, Klenk J; FARSEEING Consortium and the FARSEEING Meta Database Consensus Group. Fall detection with body-worn wearable 
sensors: a systematic review. Z Gerontol Geriatr. 2013; 46: 706-19. doi: 10.1007/s00391013-0559-8.

[12] Stack E. Falls are unintentional: Studying simulations is a waste of faking time. J Rehabil Assist Technol Eng. 2017; 4: p.2055668317732945.

[13] Chaudhuri S, Thompson H, Demiris G. Fall detection devices and their use with older adults: a systematic review. J Geriatr Phys Ther 2014; 37(4): 178-96. doi: 10.1519/JPT.0b013e3182abe779.

[14] Liang D, Ivanov K, Li H, Ning Y, Zhang Q, Wang L, Zhao G. Exploration and comparison of the pre-impact lead time of active and passive falls based on inertial wearable sensors. Biomed Mater Eng 2014; 24: 279-88. doi: 10.3233/ BME-130809.

[15] Bloch F, Gautier V, Noury N, Lundy J-E, Poujaud J, Claessens Y-E, Rigaud A-S. Evaluation under real-life conditions of a stand-alone fall detector for the elderly subjects. Ann Phys Rehabil Med. 2011; 54 (6): 391-398

[16] Podsiadlo D, Richardson S. The timed up and go: a test of basic functional mobility for frail elderly persons. JAGS. 1991; 39: 142-48.

[17] Weiss A, Herman T, Giladi N, Hausdorff JM. Objective Assessment of Fall Risk in Parkinson's Disease Using a Body-Fixed Sensor Worn for 3 Days. PLoS ONE. 2014; 9: e96675. doi:10.1371/journal.pone.0096675.

[18] Rahimia F, Bee C, Duval C, Boissy P, Edwards R, Jog M and the EMAP Group. Using Ecological Whole Body Kinematics to Evaluate Effects of Medication Adjustment in Parkinson Disease. J Parkinsons Dis. 2014; 4: 617-627 DOI 10.3233/JPD-140370.

[19] Iluz T, Gazit E, Herman T, Sprecher E, Brozgol M, Giladi N, Mirelman A, Hausdorff JM. Automated detection of missteps during community ambulation in patients with Parkinson's disease: a new approach for quantifying fall risk in the community setting. J Neuroeng Rehabil. 2014; 11: 48. DOI: 10.1186/1743-0003-11-48.

[20] Giladi N, Herman T, Reider-Groswasser II, Gurevich T, Hausdorff JM. Clinical characteristics of elderly patients with a cautious gait of unknown origin. J Neurol. 2005; 252: 300 - 06. DOI 10.1007/s00415-005-0641-2.

[21] Tsaia YJ, Lina SI. Older adults adopted more cautious gait patterns when walking in socks than barefoot. Gait Posture. 2013; 37: 88 - 92. doi 10.1016/j.gaitpost.2012.06.034. [22] Cham R, Redfern MS. Changes in gait when anticipating slippery floors. Gait Posture 2002; 15: $159-71$. 
[23] Delbaere K, Sturnieks DL, Crombez G, Lord SR. Concern About Falls Elicits Changes in Gait Parameters in Conditions of Postural Threat in Older People. J Gerontol A Biol Sci Med Sci. 2009; 64: 237-42. doi: 10.1093/gerona/gIn014.

[24] Bryant MS, Pourmoghaddam A, Thrasher A. Gait changes with walking devices in persons with Parkinson's disease. Disabil Rehabil: Assist Technol. 2012; 7: 149-52.

[25] Weiss A, Shimkin I, Giladi N, Hausdorff JM. Automated detection of near falls: algorithm development and preliminary results. BMC Research Notes. 2010; 3:62.

[26] Iluz T, Weiss A, Gazit E, Tankus A, Brozgol M, Dorfman M, Mirelman A, Giladi N, Hausdorff JM. Can a Body-Fixed Sensor Reduce Heisenberg's Uncertainty When It Comes to the Evaluation of Mobility? Effects of Aging and Fall Risk on Transitions in Daily Living. J Gerontol A Biol Sci Med Sci 2016; 71: 1459-65.

[27] Stack E, Ashburn A. Dysfunctional turning in Parkinson's disease. Disabil Rehabil. 2008; 30: 1222 - 9. doi: 10.1080/09638280701829938.

[28] Lyons JG, Heeren T, Stuver SO, Fredman L. Assessing the Agreement Between 3Meter and 6-Meter Walk Tests in 136 Community-Dwelling Older Adults. J Aging Health. 2015; 27: 594-605.

[29] Huntington Study Group. Unified Huntington's Disease Rating Scale: Reliability and Consistency. Movement Disorders. 1996; 11: $136-42$.

[30] Dion L, Malouin F, McFadyen, BJ, Richards CL. The Rise-To-Walk task for assessing mobility and locomotor co-ordination after stroke. Neurorehabil Neural Repair 2003; 17: 83 $-92$.

[31] Stack E, Ashburn A, Jupp K. Postural instability during reaching tasks in Parkinson's disease. Physiother Res Int. 2005; 10:146-53. doi: 10.1002/pri.4.

[32] Keus SHJ, Nieuwboer A, Bloem BR, Borm GF, Munneke M. Clinimetric analyses of the Modified Parkinson Activity Scale. Parkinson Rel Dis 2009; 15: 263-69. doi.org/10.1016/j.parkreldis.2008.06.003.

[33] Matias R, Paixão V, Bouça R, Ferreira JJ. A Perspective on Wearable sensor Measurements and Data science for Parkinson's Disease. Front Neurol. 2017; 8: 677. [34] Del Din S, Godfrey A, Mazzà C, Lord S, Rochester L. Free - living monitoring of Parkinson's disease: Lessons from the field. Movement Disorders. 2016; 31:1293-313. 
[35] Hubble RP, Naughton,GA., Silburn PA, Cole MH. Wearable sensor use for assessing standing balance and walking stability in people with Parkinson's disease: a systematic review. PloS One. 2015; 10: p.e0123705. doi: 10.1371/journal.pone.0123705.

[36] Pang I, Okubo Y, Sturnieks D, Lord SR, Brodie MA. Detection of Near Falls Using Wearable Devices: A Systematic Review. Journal of geriatric physical therapy 2018; 2001. doi: 10.1519/JPT.0000000000000181.

[37] Bagalà F, Becker C, Cappello A, Chiari L, Aminian K, Hausdorff JM, Zijlstra W, Klenk J. Evaluation of wearable sensors-based fall detection algorithms on real-world falls. PLoS One. 2012; 7:e37062. doi: 10.1371/journal.pone.0037062.

[38] Stack E, King R, Janko B, Burnett M, Hammersley N, Agarwal V, Hannuna S, Burrows A, Ashburn A. Could In-Home Wearable sensors Surpass Human Observation of People with Parkinson's at High Risk of Falling? An Ethnographic Study. BioMed Research International 2016; Article ID 3703745. http://dx.doi.org/10.1155/2016/3703745.

[39] Nieuwboer A, Weerdt WD, Dom R, Lesaffre E. A frequency and correlation analysis of motor deficits in Parkinson patients. Disabil Rehabil. 1998; 20: 142-50.

[40] Wood BH, Bilclough JA, Bowron A, Walker RW. Incidence and prediction of falls in Parkinson's disease: a prospective multidisciplinary study. J Neurol Neurosurg Psychiatry. 2002; 72: 721-25.

[41] Maki BE, Mcllroy WE. Control of rapid limb movements for balance recovery: agerelated changes and implications for fall prevention. Age Ageing. 2006; 35: ii12-ii18. [42] Young WR, Hollands MA . Can telling older adults where to look reduce falls? Evidence for a causal link between inappropriate visual sampling and suboptimal stepping performance. Exp Brain Res. 2010; 204: 103-13. doi 10.1007/s00221-010-2300-9. 
Table 1

Agreement on Caution and Near-falls between Ratings, by Test (ordered by \% agreement; high is best)

Subtle Instability

n

\begin{tabular}{ccc}
\hline from Video & $\begin{array}{c}\text { from Wearable } \\
\text { Sensors }\end{array}$ & n Agreed \\
\hline $3(13 \%)$ & $6(25 \%)$ & $21 / 24(88 \%)$ \\
$6(30 \%)$ & $2(10 \%)$ & $16 / 20(80 \%)$ \\
$2(20 \%)$ & 0 & $8 / 10(80 \%)$ \\
$3(23 \%)$ & 0 & $10 / 13(77 \%)$ \\
$4(36 \%)$ & $3(27 \%)$ & $8 / 11(73 \%)$ \\
$6(38 \%)$ & $5(31 \%)$ & $11 / 16(69 \%)$ \\
$10(100 \%)$ & $6(60 \%)$ & $6 / 10(60 \%)$ \\
$1(8 \%)$ & $6(46 \%)$ & $6 / 13(46 \%)$ \\
\hline $35(30 \%)$ & $28(24 \%)$ & $86(74 \%)$
\end{tabular}

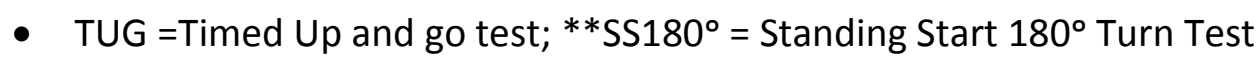


Table 2

Agreement between Video and Wearable Evaluations of Stability, $n=117$

\section{From Wearable Sensors}

\begin{tabular}{lllll} 
& & Stable & Cautious or Unstable & Total \\
\cline { 2 - 5 } From Video & Stable & $70(85 \%)$ & $12(15 \%)$ & 82 \\
\cline { 2 - 5 } & Cautious or Unstable & $19(54 \%)$ & $16(46 \%)$ & 35 \\
\cline { 2 - 5 } & Total & 89 & 28 & 117
\end{tabular}

Percentages shown are of the row total 
Table 3

Agreement on Continuous Measures (ordered by deviation from video mean; low is best)

\begin{tabular}{|c|c|c|c|c|c|c|}
\hline & \multirow[b]{3}{*}{$\mathbf{n}$} & & & \multirow{3}{*}{$\begin{array}{c}\text { Mean } \\
\text { Difference }\end{array}$} & \multicolumn{2}{|c|}{ 95\% Limits of Agreement } \\
\hline & & \multicolumn{2}{|c|}{ Mean (SD) } & & \multirow[b]{2}{*}{ Range } & \multirow{2}{*}{$\begin{array}{c}\text { As \% of } \\
\text { video mean }\end{array}$} \\
\hline & & Video & $\begin{array}{l}\text { Wearable } \\
\text { Sensors }\end{array}$ & & & \\
\hline TUG Time * & 20 & $15.4 \mathrm{~s}(7.0)$ & $16.3 \mathrm{~s}(7.0)$ & $-0.9 s(0.5)$ & $-1.9 \mathrm{~s}$ to $0.1 \mathrm{~s}$ & $13 \%$ \\
\hline 3m Walk Time & 11 & $3.7 \mathrm{~s}(1.1)$ & $3.7 \mathrm{~s}(1.3)$ & Os (0.4) & $-0.9 \mathrm{~s}$ to $0.9 \mathrm{~s}$ & $24 \%$ \\
\hline $\begin{array}{l}\text { Tandem Walk } \\
\text { Time }\end{array}$ & 10 & $10.6 \mathrm{~s}(1.6)$ & $12.0 \mathrm{~s}(1.6)$ & $-1.4 \mathrm{~s}(0.7)$ & $-2.8 \mathrm{~s}$ to $0 \mathrm{~s}$ & $27 \%$ \\
\hline 3m Walk Steps & 11 & $6.1(1.1)$ & $6.8(1.4)$ & $-0.7(0.6)$ & -1.9 to 0.5 & $31 \%$ \\
\hline SS-180 Time** & 23 & $3.0 s(1.9)$ & $2.9 s(2.0)$ & $0.1 \mathrm{~s}(0.5)$ & $-0.9 \mathrm{~s}$ to $1.1 \mathrm{~s}$ & $37 \%$ \\
\hline SS-180 Steps** & 23 & $5.1(3.3)$ & $5.2(3.4)$ & $-0.2(0.9)$ & -2.0 to 1.7 & $39 \%$ \\
\hline Sit-to-Stand Time & 25 & $2.5 \mathrm{~s}(1.4)$ & $2.7 \mathrm{~s}(1.1)$ & $-0.3 s(0.5)$ & $-1.2 \mathrm{~s}$ to $0.7 \mathrm{~s}$ & $47 \%$ \\
\hline Stand-to-Sit Time & 25 & $2.3 \mathrm{~s}(0.8)$ & $2.6 \mathrm{~s}(0.7)$ & $-0.3 s(0.4)$ & $-1.2 \mathrm{~s}$ to $0.6 \mathrm{~s}$ & $51 \%$ \\
\hline Rise-to-Walk Time & 13 & $2.8 \mathrm{~s}(2.0)$ & $2.4 \mathrm{~s}(1.2)$ & $0.4 \mathrm{~s}(0.9)$ & -1.3 to $2.2 \mathrm{~s}$ & $78 \%$ \\
\hline
\end{tabular}

- $\mathrm{TUG}=$ Timed $\mathrm{Up}$ and go test; ${ }^{* *} \mathrm{SS} 180^{\circ}=$ Standing Start $180^{\circ}$ Turn Test 
Table 4

Agreement on PAS and Other Strategies (ordered by \% agreement, high is best)

\begin{tabular}{|c|c|c|c|c|c|}
\hline & $\mathrm{n}$ & value & Video & Wearable Sensors & Agreement \\
\hline High Reach Strategy & 13 & Up-on-Toes & $8(62 \%)$ & $5(38 \%)$ & $10 / 13(77 \%)$ \\
\hline Rise-to-Walk Fluidity & 12 & Fluid & $7(58 \%)$ & $8(67 \%)$ & $9 / 12(75 \%)$ \\
\hline Low Reach Support & 10 & Used Support & 0 & $3(30 \%)$ & $7 / 10(70 \%)$ \\
\hline PAS* - TUG ** & 20 & Difficulty/hands used & $12(60 \%)$ & $18(90 \%)$ & $14 / 20(70 \%)$ \\
\hline \multirow[t]{2}{*}{ High Reach Support } & 13 & Used Support & $2(15 \%)$ & $4(31 \%)$ & 9/13 (69\%) \\
\hline & & Toward & $3(7 \%)$ & $5(11 \%)$ & \\
\hline \multirow[t]{3}{*}{ SS-180*** Turn Types } & 44 turns & Pivotal & $2(5 \%)$ & $3(7 \%)$ & $29 / 44(66 \%)$ \\
\hline & & Lateral & $13(30 \%)$ & $14(32 \%)$ & \\
\hline & & On-the-Spot & $26(59 \%)$ & $22(50 \%)$ & \\
\hline PAS - Rise-to-Walk & 11 & Difficulty/hands used & 7 (64\%) & $11(100 \%)$ & $7 / 11(64 \%)$ \\
\hline Low Reach Strategy & 13 & Squat & $2(18 \%)$ & $5(45 \%)$ & $8 / 13(62 \%)$ \\
\hline PAS - Sit-to-Stand & 25 & Difficulty/hands used & $16(64 \%)$ & $17(68 \%)$ & $14 / 25(56 \%)$ \\
\hline \multirow[t]{2}{*}{ PAS - Stand-to-Sit } & 25 & Difficulty/hands used & $15(60 \%)$ & $18(72 \%)$ & $14 / 25(56 \%)$ \\
\hline & & 0 deviations & $3(30 \%)$ & $9(90 \%)$ & \\
\hline \multirow{2}{*}{$\begin{array}{l}\text { Tandem Walk } \\
\text { Deviation }\end{array}$} & 10 & 1-3 deviations & $6(60 \%)$ & $1(10 \%)$ & $4 / 10(40 \%)$ \\
\hline & & $>3$ deviations & $1(10 \%)$ & 0 & \\
\hline
\end{tabular}

- $\mathrm{PAS}=$ Parkinson's Activity Scale; $* *$ TUG $=$ Timed Up and go test $; * *$ SS $-180^{\circ}=$ Standing Start $180^{\circ}$ Turn Test 